\title{
On Replacement Models Via A Fuzzy Set Theoretic Framework
}

\author{
Augustine O. Esogbue and Warren E. Hearnes II
}

\begin{abstract}
Uncertainty is present in virtually all replacement decisions due to unknown future events, such as revenue streams, maintenance costs, and inflation. Fuzzy sets provide a mathematical framework for explicitly incorporating imprecision into the decision making model, especially when the system involves human subjectivity. This paper illustrates the use of fuzzy sets and possibility theory to explicitly model uncertainty in replacement decisions via fuzzy variables and fuzzy numbers. In particular, a fuzzy set approach to economic life of an asset calculation as well as a finite horizon single asset replacement problem with multiple challengers is discussed. Because the use of triangular fuzzy numbers provides a compromise between computational efficiency and realistic modeling of the uncertainty, this discussion emphasizes fuzzy numbers. The algorithms used to determine the optimal replacement policy incorporate fuzzy arithmetic, dynamic programming with fuzzy rewards, the vertex method, and various ranking methods for fuzzy numbers. A brief history of replacement analysis, current conventional techniques, the basic concepts of fuzzy sets and possibility theory, and the advantages of the fuzzy generalization are also discussed.
\end{abstract}

Keywords - Replacement analysis, fuzzy sets, possibility theory, fuzzy numbers, decision making under uncertainty.

\section{Economic Decision Analysis}

Economic decision analysis is a useful tool, offering individuals and organizations the techniques to model economic decision making problems, such as maintenance and replacement decisions, and determine an optimal decision. However, the accuracy of the model determines the validity of the conclusion. In many cases, the assumption of certainty in many models is made not so much for validity but the need to obtain simpler and more readily solvable formulations. Essentially, the tradeoff is between an inaccurate but solvable model and a more accurate but potentially unsolvable one. In most real-world systems, however, there are elements of uncertainty in the process or its parameters, which may lack precise definition or precise measurement, especially when the system involves human subjectivity.

When developing a model of a system with uncertainty, the decision maker can either ignore the uncertainty, implicitly acknowledge it, or explicitly model it. Ignoring the uncertainty usually results in a deterministic model of the process with precise values for all parameters. Implicitly acknowledging the uncertainty may still result in a deterministic model in which sensitivity analysis or discount factors can be used to get an idea of how this uncertainty affects the outcome. Lastly, the decision maker can explicitly model the uncertainty using specific paradigms such as interval analysis, possibility theory, probability theory, or evidence theory [3].

The proper paradigm depends on the nature of the uncertainty. When the probabilities are specified for the out- comes, then the theory of Von Neumann and Morgenstern [40] provides the tools necessary to determine the optimal decision. However, in many cases these probabilities are neither defined nor directly attainable. Under these circumstances, other theories are needed. The most common choice is the use of subjective probability distributions and the theory of choice due to Savage [34]. However, considerable debate on the use of subjective probabilities exists and is well documented in the literature [6], [16], [23], [25], [27]. From a psychological standpoint, the methods used to elicit these subjective probabilities and the validity of the subjective probabilities themselves have been the focus of research led by Tversky and Kahneman [37], [39], [38]. Their studies show that the heuristics employed to assess probabilities and predict values can sometimes lead to "severe and systematic errors" [38].

Because humans do not think naturally in probabilistic terms, they tend to find the notions of fuzzy sets and their linguistic based approaches more user-friendly and appealing. We may view fuzzy set theory as a generalization of classical set theory since it provides us with a mathematical tool for describing sets that have no sharp transition from membership to nonmembership. Membership in a fuzzy set is defined by a generalized version of the classical indicator function called a membership function. Fuzzy sets allow the definition of vague or imprecise concepts such as "approximately 1000" where, for example, 1000 would have a membership of 1.0 and 975 a membership of 0.5 (see Figure 1). This theory has been developed and successfully applied to numerous areas such as control and decision making, engineering, and medicine. Its application to economic analysis is natural due to the uncertainty inherent in many financial and investment decisions. As noted earlier, it provides a precise mathematical language to model uncertainty due to vagueness and imprecision in events or statements describing a system. More information on fuzzy set theory, particularly fundamental concepts such as fuzzy numbers which are invoked in our presentation, is included in the Appendix.

\section{RePlacement AnAlysis}

One of the most practical and topical areas of engineering economics is replacement analysis. Mathematical models and analysis methods are used to determine the sequence of replacement decisions that provides a required service for a specified time horizon in an optimal manner. It is assumed that maintenance and replacement decisions occur on a periodic basis. The decision maker chooses from various options, such as to keep, overhaul, or perform preventive maintenance on the existing asset or replace it with 
a new/used asset. Any sequence of decisions is called a replacement policy and any sequence that optimizes some performance measure such as net present value or annual equivalent cost is an optimal replacement policy.

In replacement analysis the economic life of an asset determines the replacement cycle that gives the minimum annual equivalent cost (MAEC) of operating a single asset over an infinite horizon [35]. Dynamic programming (DP), with discounting to put more emphasis on short-term income, is an acknowledged tool for the determination of the optimal replacement policy for more general replacement models [18]. Using the DP approach some of the restrictive assumptions of the economic life method can be relaxed and still produce a computationally feasible solution algorithm. Early pioneers in the use of dynamic programming for finite horizon equipment replacement problems were Bellman and Wagner. Bellman [4], [5] was the first to formulate the replacement problem as a dynamic program. Optimal replacement policies were proposed first for the case with no technological change and the other assuming technological improvement. Bellman formulated a discounted DP version of the economic life of an asset model and determined analytically the optimal age $T$ to replace the asset. In the more challenging technological improvement version, the revenue, the upkeep costs, and the replacement costs are assumed to be functions of the date the asset is installed as well as its age with respect to installation. Wagner [41] formulated the replacement problem as a network and solved for the shortest path, which corresponded to the minimum outlay. Terborgh [36] included linear obsolescence in his formulation while Alchian [1] allowed operating revenues and operating costs to increase linearly with time. Oakford [30] modeled increasing revenues and data. Dreyfus [13] modeled technological change in revenue, maintenance, and replacement costs using bounded exponential functions. The Dynamic Replacement Economy Decision Model (DREDM) developed in [31] is a generalization of Wagner's dynamic programming model that allows for multiple challengers and timevarying parameters.

Replacement models of great interest and relevance to this research are those that model uncertainty. Dreyfus and Law [13] treat the replacement problem where determinism yields to stochasticity. Their model includes the probability of a catastrophic failure in the asset being used as well as an uncertain net operating cost that is modeled by another probability distribution. The DP algorithm determines the minimum expected cost for the process. The Stochastic Replacement Economy Decision Model (SRE) presented by Lohmann [29] is a stochastic generalization of the DREDM. The assumption that the cash flows and relationships are known with certainty was relaxed and the component cash flows are modeled as triangular probability distributions based on the decision maker's subjective judgment. The solution for this model is generated through Monte-Carlo simulation, which determines the probability that each asset is the optimal choice at time 0 as well as the probability distribution of the optimal net present value of the policy.

A tacit assumption implicit in the foregoing models is that uncertainty in the replacement decision can be fully modeled either deterministically or stochastically. This is not, however, always the case. Limiting replacement models to these two approaches either ignores the uncertainty or assumes that all uncertainty is probabilistic in nature and that the probabilistic information is fully known. Categorically classifying all uncertainty as randomness may not be reasonable or adequate.

In recent times the debate concerning the use of nonprobabilistic uncertainty, and specifically fuzzy sets, has surfaced in the area of economic analysis [3], [8], [9], [10], [11], [19], [20], [42]. The replacement decisions made at each time period are based not only on the current cash flows but also projected future cash flows of all possible assets [29]. Therefore, uncertainty in these cash flows can have a pronounced effect on the optimal replacement policy. A fuzzy set theoretic approach, as described in the sequel, may lead to more informed replacement decisions when the assumptions for a probabilistic approach are not met. For the deterministic case, a fuzzy set theoretic approach using fuzzy numbers is equivalent to multivariable sensitivity analysis and immediately provides both the deterministic optimal value and the possible range due to uncertainty.

\section{Fuzzy Concepts in CASH Flow Analysis}

Cash flows, the basic variable in replacement decisions, are used by managers and financial analysts to measure the streams of money going into and flowing out of a particular organization's operation [35]. Traditionally, cash flows are treated as either deterministic or stochastic. However, as shown in simulation studies [32], uncertain information in estimating these cash flows can limit the value of the analysis. Errors in deterministic cash flow estimations can skew the results of the analysis. Similarly, the use of subjective probability distributions is of concern since they generally cannot be verified, and the required historical information for generating frequency-based probability distributions is not always available.

The fundamental types of uncertainty, nonspecificity, fuzziness, and strife, are examined by Klir and Yuan in [24]. Uncertainty occurs in replacement and maintenance decisions in various ways. Of particular interest are nonspecificity and fuzziness which may factor into the estimates of the aggregate cash flows, the purchase prices/salvage values, the minimum attractive rate of return (MARR), or the physical lifetimes of the assets. This is especially true when these variables are based on the estimates provided by experts via the use of such natural language statements as "approximately $\$ 1000 "$. Using fuzzy variables one can represent this vagueness and imprecision. In this presentation, however, these vague quantities will be represented using triangular fuzzy numbers (TFNs).

Several basic concepts of fuzzy sets applicable to the foregoing forms of uncertainty are fuzzy sets, $\alpha$-level sets, convexity, and triangular fuzzy numbers. For completeness, these definitions and the foundations of fuzzy numbers and 
possibility theory are briefly reviewed in the Appendix. The reader is referred to [45], [46] for a more complete review. Fuzzy numbers are fuzzy sets defined on the set of real numbers, generally used to represent vague expressions, such as "about 20" or "approximately 1000", used often in the description of uncertain economic decision systems. Triangular fuzzy numbers are a special type fuzzy number which simplify the arithmetic operations considerably and are used in the models developed in this research. Figure 1 is a triangular fuzzy number representation of the expression "approximately 1000." A triangular fuzzy num-

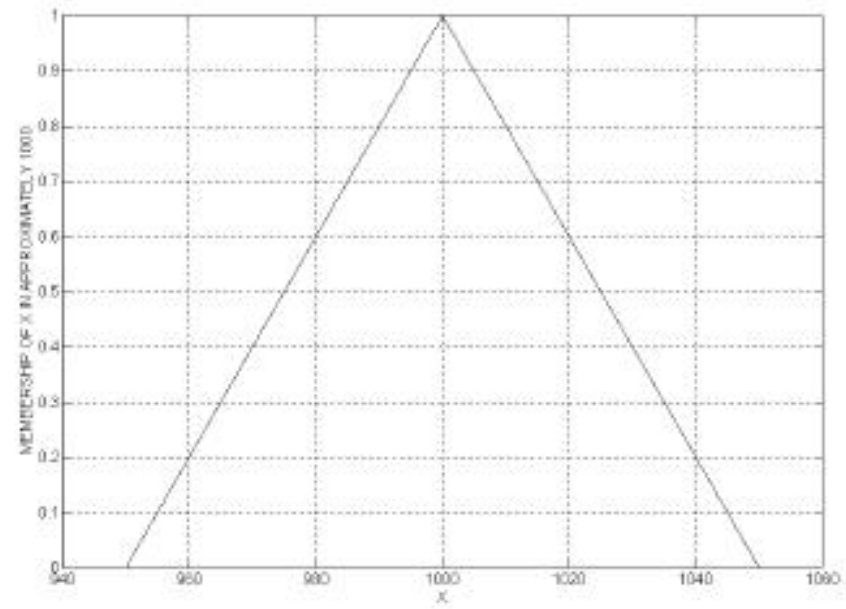

Fig. 1. TFN representing the expression "approximately 1000."

$\operatorname{ber}(\mathrm{TFN})$ is a fuzzy number $\tilde{M}_{T}=[l, m, r]$ with the membership function

$$
\mu_{\tilde{M}_{T}}=\left\{\begin{array}{cl}
(1 /(m-l))(x-l), & \text { for } x \in(l, m] \\
(1 /(r-m))(r-x), & \text { for } x \in(m, r) \\
0, & \text { otherwise }
\end{array}\right.
$$

\section{A. Nonprobabilistic Methods in Cash Flow Analysis}

Research into fuzzy versions of cash flows began with Ward [42], defining them as trapezoidal fuzzy numbers and solving a fuzzy present worth problem. Buckley [8] used fuzzy numbers to develop fuzzy net present value (FPV) and fuzzy net future value (FFV) with fuzzy interest rates over a period of $n$ years where $n$ may also be fuzzy set. Buckley developed fuzzy equivalents to continuous interest payments, the effective rate of interest, and regular annuities as well. Restricting the fuzzy cash flows to positive fuzzy numbers allows the multiplication operation to be distributive over addition. The fuzzy number of time periods produces nonlinearities that make computations more complex. Li Calzi [28] provided an axiomatic development for the fuzzy extension of financial mathematics with a desire to maintain consistency in the calculations. He examined two classes of fuzzy quantities, compact fuzzy intervals and invertible fuzzy intervals, and proved general theorems regarding consistency.

Two of the most recent and practical applications of nonprobabilistic uncertainty to economic analysis are given in [10], [11]. Choobineh and Behrens [11] call attention to the use of intervals and possibility theory in economic analysis. The weak distributivity of interval arithmetic is noted, but a technique called the vertex method [12] is utilized to bypass this problem in interval and fuzzy arithmetic. Their approach to modeling cash flows as fuzzy intervals is similar to Ward's. Chiu and Park [10] use fuzzy numbers in cash flow analysis and provide a good survey of the major methods for ranking mutually exclusive fuzzy projects. The cash flows are modeled as triangular fuzzy numbers and the linear approximation to the product of two triangular fuzzy numbers is investigated. The present worth of a fuzzy project is also examined. Their resultant formulation of a fuzzy present worth is

$$
P W=\sum_{t=0}^{n}\left\{\frac{P_{t}}{\prod_{s=0}^{t}\left(1+R_{s}\right)}\right\}
$$

where $P_{t}$ is a positive or negative TFN representing the cash flow at the end of time $t, n$ is the number of evaluation periods, and $R_{s}$ is the nonnegative TFN representing the discount rate at the end of time $s$. Extending these ideas to replacement analysis, Hearnes [20] formulated fuzzy versions of the economic life of an asset model and the finite single asset replacement problem. This work is used as a point of departure for the discussions that follow.

\section{B. Fuzzy Arithmetic and Interval Analysis}

Fuzzy numbers represent vague notions of precise quantities. It is essential to be able to perform algebraic operations on them. Fuzzy arithmetic is based on the extension principle introduced by Zadeh in 1975 [44]. The arithmetic operations of addition, subtraction, multiplication, and division developed in [14] and [15] are particularly useful when modeling and analyzing cash flows. The algebraic operations for TFNs are specifically reviewed. The choice of TFNs is in part due to their simplified algebraic operations. However, he set of TFNs is not closed under the operations of multiplication and division. The effect of using a linear (TFN) approximation, which is studied thoroughly in [22], is not significant. The TFN approximation to the multiplication operation is used in the following models for computational simplicity.

Fuzzy numbers are a family of nested intervals [11] which correspond to levels of "confidence" by the decision maker and therefore are closely related to interval analysis. However, like interval arithmetic, the multiplication operation for fuzzy numbers is only weakly distributive over addition. This presents a problem when modeling with fuzzy numbers since the outcome can depend on the form of the equation used. There are some special cases, however, where the multiplication operation is distributive over addition. If $\tilde{M}_{1}, \tilde{M}_{2}, \tilde{M}_{3}$ are fuzzy numbers, the multiplication operation is distributive over addition (i.e., $\tilde{M}_{1} \cdot\left(\tilde{M}_{2}+\tilde{M}_{3}\right)=$ $\left.\tilde{M}_{1} \cdot \tilde{M}_{2}+\tilde{M}_{1} \cdot \tilde{M}_{3}\right)$ when $[15]$ :

1. $\tilde{M}_{1}$ is a real number-i.e., scalar multiplication is distributive over addition.

2. $\tilde{M}_{2}$ and $\tilde{M}_{3}$ are both positive or both negative.

3. $\tilde{M}_{2}=-\tilde{M}_{2}$ and $\tilde{M}_{3}=-\tilde{M}_{3}$. 
When conditions (1) - (3) are not met, a procedure called the vertex method [12] preserves the distributivity of multiplication over addition. The price, however, is an exponential increase in the number of computations.

\section{Some Sources of Uncertainty in Replacement Analysis}

Several aspects of replacement analysis contain imprecision and vagueness that warrant further discussion. We postulate that some of these variables, such as the physical lifetime of an asset, aggregate cash flow estimates, and the MARR, significantly impact the optimal replacement policy.

The physical lifetime of some assets may not be known with certainty, yet this is tacitly assumed and treated as deterministic in many models. In some situations, if enough information is known, then it is appropriate to treat it probabilistically. However, in cases where the asset is a new technology or a new model, this information is not generally available and such an approach must be considered suspect. In this case, it is instructive to treat the uncertainty in this variable through the use of fuzzy DP models but particularly those that allow stochastic or fuzzy termination times [8], [21]. For example, for an older asset the historical information about failures may be known and a probability distribution for failure can be derived. However, for an asset with new technology estimate of physical lifetime may be a fuzzy set such as "about 5 years" or "more or less 10 years". In each of these cases, the decision space has an uncertain boundary that affects the overall decision policy. Stochastic and fuzzy DP [17] provide methods for dealing with this type of uncertainty. Similarly, there may be uncertainty in the actual horizon $N$ of the project which may be either stochastic or fuzzy. For example, the project duration of asset life may be determined if the state of the asset reaches some imprecisely definable point.

Another source of uncertainty in replacement and maintenance decisions is the estimation of the aggregate cash flow for each time period. In previous models, aggregate cash flows were treated as either deterministic or stochastic variables, but errors in these can lead to skewed analysis [32]. Subjective probability distributions generally cannot be verified, while the required historical information for generating frequency-based probability distributions is not generally available. In these cases, it may be more appropriate to define the aggregate cash flows as possibility distributions based on a decision maker's opinion or expert judgment.

The minimum attractive rate of return (MARR), which is usually used for project evaluation and comparison, is also another variable which may realistically possess forms of uncertainty [35]. However, in classical approaches this either is not addressed or is erroneously assumed to be well known or deterministic. The selection of the proper MARR plays an important role in the outcome of the maintenance and replacement decisions. There are a number of ways to determine a corporation's MARR, such as the use of the Delphi method involving its directors or some chosen mathematical formula. However, the MARR can be investment or management dependent. Because of the uncertainties characteristic of investment and management decision processes, it is inevitable that any MARR thus determined is imprecise or fraught with uncertainties. Variation in the MARR and its effect on the optimal policy are vital pieces of information to decision makers. These may be better modeled as a fuzzy variable or fuzzy number.

A number of engineering economic studies discuss the incorporation of inflation and inflation rate in their models. It is tacitly assumed or conceded that the measurement of this variable is precise. This, however, is not the case. We know that there is a considerable degree of uncertainty due to the way that it is measured. For example, the Consumer Price Index presently used by the government of the United States of America is now under review due to the concern expressed by certain economists that the "basket" of goods and services it uses may not accurately reflect the true inflation (see, for example, [33]). The lack of specificity or precision involved in the measurement of the inflation rate may necessitate the injection of fuzzy modeling such as the use of fuzzy numbers to represent it.

\section{Economic Life of an Asset Model}

In some replacement decisions, an asset is required for a long period of time. In these cases an infinite horizon can be assumed and the decision variable becomes the life of the asset, commonly called the economic life of an asset. The chosen replacement cycle is the cycle corresponding to the minimum annual equivalent (AE) cost of owning and operating the asset [35]. An infinite sequence of replacements and stationary cash flows (with respect to installation time) is assumed.

The general deterministic $n$-period replacement cycle gives the following $A E$ cost:

$$
\begin{aligned}
A E_{n}(i)= & \left(P-S_{n}\right)(A / P, i, n)+S_{n} i \\
& +(A / P, i, n) \sum_{n^{\prime}=1}^{n}\left(C_{n^{\prime}}\left(P / F, i, n^{\prime}\right)\right)
\end{aligned}
$$

where

$$
\begin{aligned}
i \equiv & \text { minimum attractive rate of } \\
& \text { return (MARR) } \\
P \equiv & \text { initial purchase price } \\
S_{n} \equiv & \text { salvage value at end of period } n \\
C_{n^{\prime}} \equiv & \text { aggregate cash outflow at end of } \\
& \text { period } n^{\prime} \\
(A / P, i, n) \equiv & \text { capital recovery factor } \\
(P / F, i, n) \equiv & \text { present worth factor }
\end{aligned}
$$

Two significant factors determining the optimal replacement cycle are the aggregate cash flows at each time period and the MARR. The MARR is set by the organization and is considered a crisp (deterministic) number in the model. The future aggregate cash flows and salvage values, however, are a source of considerable uncertainty and are modeled as triangular fuzzy numbers. These parameters are represented as fuzzy versions of their original counterparts by $\tilde{C}_{n^{\prime}}$ and $\tilde{S}_{n}$, respectively. The decision maker determines a best, worst, and most likely estimate 
for each. This method of elicitation is quick and has been used previously in replacement analysis [29].

Generalizing to consider fuzzy cash flows and salvage values, one obtains the Possibilistic Economic Life of an Asset Model (PELAM) [20]. The fuzzy economic life of an asset is defined as the replacement cycle, $n$, corresponding to the minimum fuzzy $A E(F A E)$ of all possible replacement cycles. The traditional model in Equation 3 is manipulated into a proper representation - i.e., all fuzzy numbers appear only once in the equation:

$$
\begin{aligned}
F A E_{n}(i)= & P(A / P, i, n)+\tilde{S}_{n}(i-(A / P, i, n)) \\
& +(A / P, i, n) \sum_{n^{\prime}=1}^{n}\left(\tilde{C}_{n^{\prime}}\left(P / F, i, n^{\prime}\right)\right)
\end{aligned}
$$

where

$$
\begin{gathered}
\tilde{S}_{n} \equiv \begin{array}{l}
\text { TFN representing the "salvage value at } \\
\text { end of period } n "
\end{array} \\
\tilde{C}_{n^{\prime}} \equiv \begin{array}{l}
\text { TFN representing the "aggregate cash } \\
\text { flow at end of period } n^{\prime \prime}
\end{array}
\end{gathered}
$$

The operations used in PELAM are scalar multiplication, addition, and subtraction. Therefore, the use of TFNs to model the cash flows gives TFNs as a result. However, if the MARR is also modeled as a fuzzy number the result is not a TFN and the linear approximation to TFN multiplication and the vertex method must be used.

\section{An Example Problem}

An asset to perform service A is required by XYZ Corporation indefinitely. Asset B can be purchased for $\$ 50$ (all dollar amounts are in thousands) and has a physical life of 5 years. The aggregate cash flows (operating costs operating revenues) for each year of the life of asset B are $\$ 3, \$ 4, \$ 6, \$ 10$, and $\$ 12$, respectively, for $n=1, \ldots, 5$. If the asset is sold at the end of the year, its salvage value is $\$ 35, \$ 30, \$ 27, \$ 23$, and $\$ 20$, respectively, for $n=1, \ldots, 5$. Assume a MARR of $10 \%$. Determine the economic life of Asset B.

The assumption of certainty in future cash flows is unrealistic, except in some cases such as when the asset is covered by a service contract. Likewise, future salvage values are dependent on the state of the equipment at that time, possible technological breakthroughs that have occurred, and numerous other uncertain events. The deterministic data is treated as the "most likely" estimates. The local expert or decision maker provides additional information, namely the "best" and "worst" estimates:

Being pessimistic, the decision maker believes that the cash flows (operating costs - revenues) might be much higher than the "most likely" estimates, and the salvage values might be much lower. Therefore the high estimates for the cash flows are $\$ 5, \$ 7, \$ 10, \$ 15$, and $\$ 18$, respectively, for $n=1, \ldots, 5$. The low estimates remain near the "most likely" estimates $-\$ 2, \$ 3, \$ 5, \$ 8$, and $\$ 10$. The salvage values high estimates are $\$ 38, \$ 32, \$ 29, \$ 27$, and $\$ 25$. The low estimates are $\$ 32, \$ 24, \$ 21, \$ 18$, and $\$ 15$.
Table I gives the $F A E$ costs for each replacement cycle, $n=1, \ldots, 5$ while Figure 2 gives a graphical representation. Of these, the "minimum" must be chosen. Comparing al-

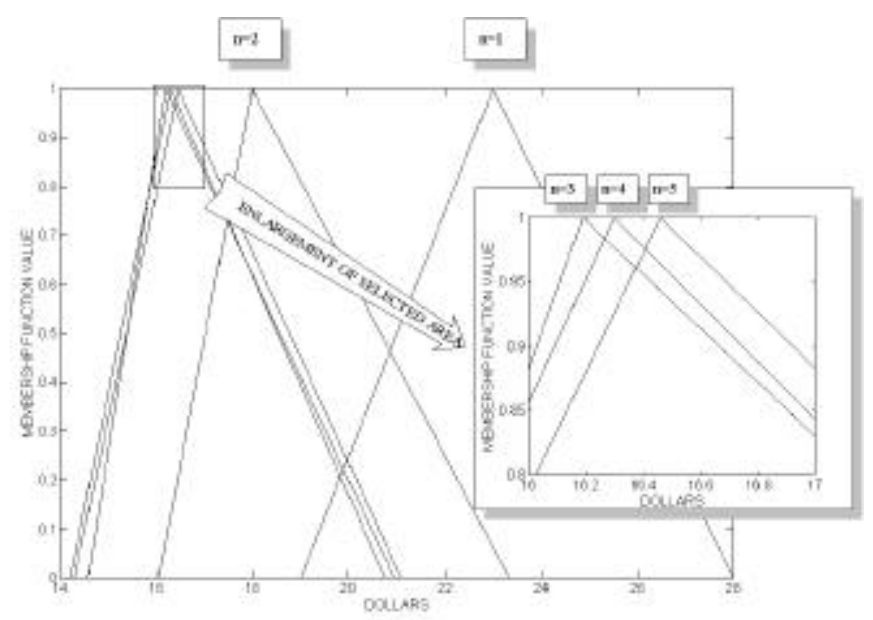

Fig. 2. TFNs representing the annual equivalent costs for the five possible replacement cycles for the example problem.

ternatives described by fuzzy numbers requires a ranking method. All ranking methods reported in the literature suffer from some pathological examples where the result is counterintuitive [7], [10]. The rankings of selected methods are given in Table II below. Note that the rankings are not all in agreement as shown in Table II. However, several of them do agree with each other (Chiu and Park, Choobineh and Behrens, and Kaufmann and Gupta). All three of these methods use a ranking function based on the $l, m$, and $r$ values of the TFN. In the remainder of this paper, the Kaufmann and Gupta method is used as the preferred ranking function.

\begin{tabular}{||c||c||}
\hline $\begin{array}{c}\text { Replacement } \\
\text { Cycle (Years) }\end{array}$ & $\begin{array}{c}\text { Fuzzy } A E \text { Cost } \\
\text { of Replacement Cycle }\end{array}$ \\
\hline \hline 1 & {$[19.00,23.00,28.00]$} \\
\hline 2 & {$[16.05,18.00,23.33]$} \\
\hline 3 & {$[14.58,16.19,20.94]$} \\
\hline 4 & {$[14.22,16.30,20.75]$} \\
\hline 5 & {$[14.30,16.46,21.09]$} \\
\hline
\end{tabular}

TABLE I

Results of PELAM for Example Problem.

The Kaufmann and Gupta method is a hierarchical test. It can be described as follows: Let $\tilde{M}_{1}=\left[l_{1}, m_{1}, r_{1}\right]$ and $\tilde{M}_{2}=\left[l_{2}, m_{2}, r_{2}\right]$ be two different TFNs.

TEST 1: Compare the ordinary numbers:

- $\operatorname{IF}\left(l_{1}+2 m_{1}+r_{1}\right)<\left(l_{2}+2 m_{2}+r_{2}\right)$ THEN $\tilde{M}_{1}<\tilde{M}_{2}$

- ELSE IF $\left(l_{1}+2 m_{1}+r_{1}\right)>\left(l_{2}+2 m_{2}+r_{2}\right)$ THEN $\tilde{M}_{1}>\tilde{M}_{2}$

- ELSE go to TEST 2.

TEST 2: Compare the modal values:

- IF $m_{1}<m_{2}$ THEN $\tilde{M}_{1}<\tilde{M}_{2}$

- ELSE IF $m_{1}>m_{2}$ THEN $\tilde{M}_{1}>\tilde{M}_{2}$

- ELSE go to TEST 3.

TEST 3: Compare the divergence: 


\begin{tabular}{||c||}
\hline Ranking Method with Ranking \\
\hline \hline Adamo, $\alpha=0.9$ \\
$F A E_{3} \sim F A E_{4} \sim F A E_{5}<F A E_{2}<F A E_{1}$ \\
\hline \hline Chang \\
$F A E_{3}<F A E_{4}<F A E_{5}<F A E_{2}<F A E_{1}$ \\
\hline \hline Chiu and Park, $w=0.3$ \\
$F A E_{4}<F A E_{3}<F A E_{5}<F A E_{2}<F A E_{1}$ \\
\hline \hline Choobineh and Behrens \\
$F A E_{4}<F A E_{3}<F A E_{5}<F A E_{2}<F A E_{1}$ \\
\hline \hline Kaufmann and Gupta \\
$F A E_{4}<F A E_{3}<F A E_{5}<F A E_{2}<F A E_{1}$ \\
\hline \hline Traditional model \\
\hline \hline$A E_{3}<A E_{4}<A E_{5}<A E_{2}<A E_{1}$ \\
\hline \hline
\end{tabular}

TABLE II

Ranking of FAE Costs in Example Problem by Various Methods.

- IF $\left(r_{1}-l_{1}\right)<\left(r_{2}-l_{2}\right)$ THEN $\tilde{M}_{1}<\tilde{M}_{2}$

- ELSE $\tilde{M}_{1}>\tilde{M}_{2}$.

This method is usually used because (1) it is relatively easy to compute and (2) it always chooses a maximum when the two TFNs are not equal. The latter property is especially important in models based on dynamic programming where a unique optimal value at each stage is desired.

\section{The Optimal Replacement Cycle for the Example Problem}

We now show the determination of the optimal replacement policy for the example problem. Using the Kaufmann and Gupta ranking method for reasons discussed above, the optimal replacement cycle for Asset B is 4 years with a fuzzy annual equivalent cost of $[14.22,16.30,20.75]$. The modal values of the TFNs for each replacement cycle correspond to the deterministic $A E$ costs from the traditional economic life of an asset model. Therefore, the traditional optimal replacement cycle is immediately available from the fuzzy solution, and the fuzzy solution using fuzzy numbers is equivalent to performing sensitivity analysis on all uncertain variables. It is also interesting to note that using the traditional model the optimal replacement cycle is 3 years, which is different from the 4 years determined by PELAM. Additionally, PELAM determines the optimal replacement cycle based on the decision maker's estimates of the uncertainty and therefore provides a more informative answer than the traditional model.

\section{General Single Asset Replacement}

When a service is required for only a finite period or the aggregate cash flows are nonstationary with respect to installation time, a more general approach than PELAM is needed. The general single asset replacement problem is widely studied in the literature [2], [13], [29], [31]. It may be defined as follows:

$$
\begin{aligned}
a_{n} \equiv & \text { asset in use at time period } n \\
N \equiv & \text { number of time periods that service } \\
& \text { is required } \\
A_{n} \equiv & \begin{array}{l}
\text { number of challenging assets at time } \\
\text { period } n
\end{array}
\end{aligned}
$$

The time periods $n=0,1, \ldots, N$ represent the periodic replacement decisions. If $N$ is finite then the problem is a finite horizon replacement problem, and if $N$ is infinite then the problem is an infinite horizon problem. The existing asset is known as the defender and can only be placed into service at period 0 . The $A_{0}$ assets available for replacement at time 0 are known as current challengers, and the $A_{n}$ assets available at future periods are known as $f u$ ture challengers. For each period $n$ in the lifetime of each asset, there are three component cash flows describing the installation cost and/or salvage value, operating costs, and operating revenues at period $n$. The component cash flows of the future challengers are related to the corresponding component cash flow of a current challenger by a scalar function $f(a, n, C)$ where $n$ is the time period in which asset $a$ is installed and $C \in 1,2,3$ represents the respective component cash flow. This function allows for the modeling of inflation, technological improvements, and other timedependent effects on cash flows. For example, to model a crisp $3 \%$ inflation rate, then $f(a, n, C) \equiv 1.03^{n}$ for all $a, C$. Other more complicated variations can be defined to model a wide range of factors. The component cash flows and relation functions are either known with certainty or estimated by the decision maker and may also be a fuzzy variable. The NPV of any sequence of cash flows $\left\{F_{0}, F_{1}, \ldots, F_{N}\right\}$ received at time periods $0,1, \ldots, n$ with respect to MARR $i$ is

$$
\sum_{n=0}^{N} \frac{F_{j}}{(1+i)^{n}} .
$$

The problem is to find the sequence(s) of keep/replace decisions that maximizes $N P V_{i}$, the net present value given some MARR $i$.

The Possibilistic Model for Single Asset Replacement via dynamic programming (PMSAR) [20] is a generalization of SREDM in [31] to allow for fuzzy parameters such as aggregate cash flows, inflation, or technological change. SREDM used the "best", "worst", and "most likely" estimates of the parameters, as in PERT analysis, to create triangular probability distributions. Using those probability distributions, Monte-Carlo simulation provided estimates of the probability of each asset being the optimal choice at period 0 . Under such conditions of estimating the distributions, it is arguable that a possibility theory approach is more appropriate. Like PELAM, PMSAR uses TFNs for cash flows. However, there is also the possibility of having uncertainty in technological improvements, inflation, or other aspects of the future challengers, and this is modeled as a TFN through a relation function $f$. The solution technique is a forward dynamic program that uses the Kaufmann and Gupta ranking method to determine the optimal decision and functional equation value at each time period. The problem is to find the sequence(s) of keep/replace decisions that maximizes $F P V_{i}$, the fuzzy net present value given some MARR $i$.

We now present a fuzzy analog of the SREDM model. For ease of exposition, we define the following variables of the model: 
1. Let $k$ be the state of the system, $k=1,2, \ldots, N$, which represents the number of periods of required service.

2. There are two decision variables: (1) $n$, the period to place an asset into service and (2) $a$, the asset to place into service.

3. The immediate reward $r_{i}(a, n, k)$ is the $F P V_{i}$ generated by placing asset $a$ into service at period $n$ and keeping it in service until period $k$.

4. The transition function $\tau(a, n, k)$ for placing asset $a$ into service at time $n$ for the remaining $k-n$ time periods is $\tau(a, n, k)=n$.

We define the function $F P V_{i}(a, n, C, k)$ as the fuzzy net present value with respect to MARR $i$ of the installation cost and/or salvage value, operating revenues, and operating costs for $C=1,2,3$, respectively, of placing asset $a$ into service at period $n$ for the remaining $k-n$ time periods. Collectively, the aggregate fuzzy net present value for all three component cash flows is

$$
F P V_{i}(a, n, k)=\sum_{C=1}^{3} F P V_{i}(a, n, C, k) .
$$

Furthermore, define the functional $F P V_{i}^{\star}(k)$ for this process as the value obtained using an optimal replacement policy from state 0 to state $k$. Invoking Bellman's Principle of Optimality results in the following functional equation of a forward dynamic program:

$$
F P V_{i}^{\star}(k)=\max _{n, a}\left\{r_{i}(a, n, k)+F P V_{i}^{\star}(n)\right\}
$$

where

$$
n \in\{0,1, \ldots, k-1\}
$$

and

$$
a \in \begin{cases}\left\{0,1, \ldots, A_{0}\right\} & \text { if } n=0 \\ \left\{1,2, \ldots, A_{n}\right\} & \text { if } n>0\end{cases}
$$

A boundary condition of $F P V_{i}^{\star}(0)=[0,0,0]$ is assigned. The max operation is performed through the Kaufmann and Gupta ranking method on TFNs. Defining $L_{a}$ as the physical lifetime, in time periods, of asset $a$ gives:

$$
r(a, n, k)= \begin{cases}F P V_{i}(a, n, k) & \text { if } k-n \leq L_{a} \\ -M & \text { otherwise }\end{cases}
$$

where $M \gg 0$ is some sufficiently large number.

Relating the parameters of future challengers to the parameters of current challengers via a fuzzy relation function $f(a, n, C)$ is a desirable feature since there may exist considerable uncertainty of the nonprobabilistic nature in future events. This addition is not without its price, however. The model requires multiplication of two fuzzy numbers, which is only weakly distributive and is not closed over TFNs. This problem may be readily circumvented by the adroit use of the vertex method [12] and a TFN approximation to the product of two TFNs [22].

\section{An Example Problem}

Let us now consider an adaptation of a replacement problem discussed by Lohmann [31] in which we specifically incorporate fuzzy uncertainty and use it as a vehicle for illustrating our point.

Three current challengers, $A_{n}=1,2,3$ for $n=1, \ldots, 15$, can replace the defender, $a=0$. The time horizon of $N=15$ years is established. Each challenger has a physical lifetime of 5 years. The defender has a remaining life of 3 years. For capital transfers, the cash flow at period 0 is the purchase cost and the cash flows at periods $n>0$ are salvage values. The component cash flows for the most likely estimates plus or minus a percentage are listed in Table III. Assume a MARR of $10 \%$. Suppose the tax rate, $d$, is $50 \%$ and MACRS depreciation tables for a 7 -year recovery period, [14.29\%, 24.49\%, 17.49\%, 12.49\%, 8.92\%, $8.92 \%, 8.92 \%, 4.46 \%$ ], are utilized in the determination of depreciation tax shield.

\begin{tabular}{||c||c||c|c|c||}
\hline$a$ & $n$ & $C=1$ & $C=2$ & $C=3$ \\
\hline \hline 0 & 0 & 5423 & 0 & 0 \\
\hline & 1 & $4194 \pm 5 \%$ & $-15791 \pm 5 \%$ & $19200 \pm 5 \%$ \\
\hline & 2 & $3355 \pm 5 \%$ & $-17685 \pm 5 \%$ & $19200 \pm 5 \%$ \\
\hline & 3 & $2684 \pm 10 \%$ & $-19808 \pm 10 \%$ & $19200 \pm 10 \%$ \\
\hline \hline 1 & 0 & 20000 & 0 & 0 \\
\hline & 1 & $16100 \pm 5 \%$ & $-8000 \pm 5 \%$ & $19200 \pm 5 \%$ \\
\hline & 2 & $13200 \pm 5 \%$ & $-8960 \pm 5 \%$ & $19200 \pm 5 \%$ \\
\hline & 3 & $10840 \pm 10 \%$ & $-10035 \pm 10 \%$ & $19200 \pm 10 \%$ \\
\hline & 4 & $8192 \pm 10 \%$ & $-11239 \pm 10 \%$ & $19200 \pm 10 \%$ \\
\hline & 5 & $6554 \pm 10 \%$ & $-12588 \pm 10 \%$ & $19200 \pm 10 \%$ \\
\hline \hline 2 & 0 & 21000 & 0 & 0 \\
\hline & 1 & $16800 \pm 5 \%$ & $-7500 \pm 5 \%$ & $19200 \pm 5 \%$ \\
\hline & 2 & $13440 \pm 5 \%$ & $-8400 \pm 5 \%$ & $19200 \pm 5 \%$ \\
\hline & 3 & $10252 \pm 10 \%$ & $-9408 \pm 10 \%$ & $19200 \pm 10 \%$ \\
\hline & 4 & $8602 \pm 10 \%$ & $-10537 \pm 10 \%$ & $19200 \pm 10 \%$ \\
\hline & 5 & $6881 \pm 10 \%$ & $-11801 \pm 10 \%$ & $19200 \pm 10 \%$ \\
\hline \hline 3 & 0 & 22000 & 0 & 0 \\
\hline & 1 & $17600 \pm 25 \%$ & $-7250 \pm 25 \%$ & $19200 \pm 5 \%$ \\
\hline & 2 & $14080 \pm 25 \%$ & $-8120 \pm 25 \%$ & $19200 \pm 5 \%$ \\
\hline & 3 & $11264 \pm 25 \%$ & $-9094 \pm 25 \%$ & $19200 \pm 10 \%$ \\
\hline & 4 & $9011 \pm 25 \%$ & $-10186 \pm 25 \%$ & $19200 \pm 10 \%$ \\
\hline & 5 & $7209 \pm 25 \%$ & $-11408 \pm 25 \%$ & $19200 \pm 10 \%$ \\
\hline & & \multicolumn{5}{|c||}{ TABLE III } & \\
& & \multicolumn{5}{|c||}{ DATA FOR EXAMPLE PROBLEM. }
\end{tabular}

The optimal sequence of decisions is determined via forward dynamic programming with rewards modeled as fuzzy numbers. Four primary cash flows create the installation cost and/or salvage value component cash flow (see Figure 3):

- Purchase cost of the asset: $\tilde{P}$.

- Tax on capital gains at the sale of the asset at time $k-n$ :

$$
d \cdot\left(\tilde{S}_{k-n}-\tilde{B}_{k-n}\right)
$$

where the book-value $\tilde{B}$ is

$$
\tilde{B}=\tilde{P}\left(1-\sum_{j=1}^{k-n} \operatorname{MACRS}_{j}\right)
$$




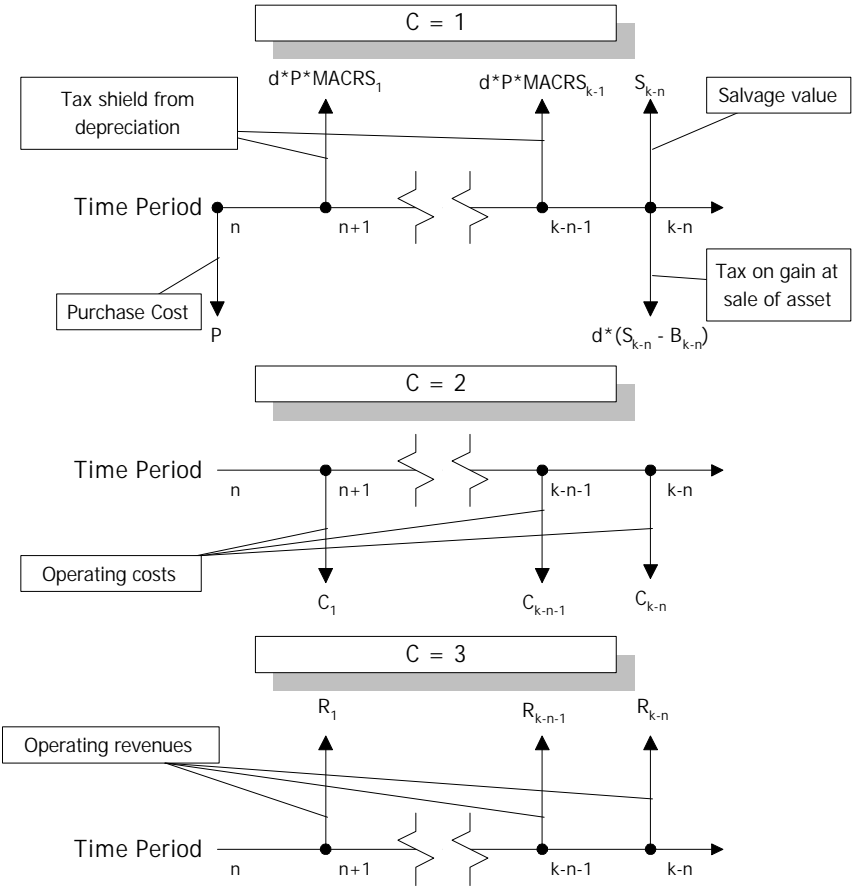

Fig. 3. Standard cash flow diagrams for each component cash flow in PSARM.

- Tax shield from depreciation of the asset during each time period:

$$
d \cdot \tilde{P} \cdot \operatorname{MACRS}_{k-n-j}
$$

for $j=0, \ldots, k-n-1$.

- Salvage value of the asset at time $k-n: \tilde{S}_{k-n}$.

The operating costs component cash flow consists of a single cash outflow representing after-tax operating costs: $\tilde{C}_{j}$ for $j=1, \ldots, k-n$. Similarly, the operating revenues component cash flow consists of a single cash inflow representing after-tax operating revenues: $\tilde{R}_{j}$ for $j=1, \ldots, k-n$. Therefore, $F P V_{i}(a, n, 1, k)$ is

$$
\frac{-\tilde{P}+\frac{\tilde{S}_{k-n}}{(1+i)^{k-n}}+d \tilde{P} \sum_{j=1}^{k-n} \frac{\mathrm{MACRS}_{j}}{(1+i)^{j}}-\frac{d\left(\tilde{S}_{k-n}-\tilde{B}_{k-n}\right)}{(1+i)^{k-n}}}{(1+i)^{n}},
$$

$F P V_{i}(a, n, 2, k)$ is

$$
-\frac{(1-d) \sum_{j=1}^{k-n} \frac{\tilde{C}_{j}}{(1+i)^{j}}}{(1+i)^{n}}
$$

and $F P V_{i}(a, n, 3, k)$ is

$$
\frac{(1-d) \sum_{j=1}^{k-n} \frac{\tilde{R}_{j}}{(1+i)^{j}}}{(1+i)^{n}} .
$$

Using the data from Table III, the calculations for $a=$ $n=0, k=1$ are as follows:

$$
\begin{aligned}
& F P V_{0.1}(0,0,1,1)=[-1146.95,-1051.64,-956.32] \\
& F P V_{0.1}(0,0,2,1)=[-7536.61,-7177.73,-6818.84] \\
& F P V_{0.1}(0,0,3,1)=[8290.91,8727.27,9163.64]
\end{aligned}
$$

which yields

$$
F P V_{0.1}(0,0,1)=[-392.66,497.91,1388.48] .
$$

Similar calculations for $a=1,2,3$ yield

$$
\begin{aligned}
& F P V_{0.1}(1,0,1)=[515.91,1500.00,2484.09] \\
& F P V_{0.1}(2,0,1)=[511.36,1500,2488.64] \\
& F P V_{0.1}(3,0,1)=[-228.41,1431.82,3092.04] .
\end{aligned}
$$

Note that the salvage and purchase values and functional relation (if used) appear more than once in the $F P V_{i}$ calculations for $C=1$, therefore the vertex method is used to generate the correct $F P V_{i}$. This entails computing deterministically the problem for the modal (most likely values) as well as an additional $2^{3}$ times for all the possible combinations of "best" and "worst" estimates for these parameters. Thus, Equation 7 gives the following for a one-year horizon, $k=1$ :

$$
\begin{aligned}
F P V_{.1}^{\star}(1) & =\max _{n, a}\left\{F P V_{.1}(a, n, 1)+F P V_{.1}^{\star}(0)\right\} \\
& =[515.91,1500.00,2484.09] \text { for } a=1 .
\end{aligned}
$$

The same calculations are performed for the remaining stages, up to $k=N$, recording both the functional equation value and the optimal decision for each stage as in Table IV.

\begin{tabular}{||c|c|c||}
\hline$N$ & $\begin{array}{c}\text { Optimal Policy } \\
\text { (asset, time installed) }\end{array}$ & $F P V_{0.1}^{\star}(N)$ \\
\hline \hline 1 & $(1,0)$ & {$[515.91,1500.00,2484.09]$} \\
\hline 2 & $(1,0)$ & {$[1686.70,3159.42,4632.15]$} \\
\hline 3 & $(1,0)$ & {$[2054.50,4759.95,7465.39]$} \\
\hline 4 & $(2,0)$ & {$[2799.62,6360.66,9921.71]$} \\
\hline 5 & $(3,0)$ & {$[413.48,7715.23,15017.00]$} \\
\hline 6 & $(3,0)(2,5)$ & {$[731.00,8646.61,16562.20]$} \\
\hline 7 & $(3,0)(1,5)$ & {$[1460.79,9676.98,17893.20]$} \\
\hline 8 & $(2,0)(2,4)$ & {$[4711.79,10705.10,16698.40]$} \\
\hline 9 & $(3,0)(2,5)$ & {$[2151.82,11664.70,21177.60]$} \\
\hline 10 & $(3,0)(3,5)$ & {$[670.22,12505.80,24341.40]$} \\
\hline 11 & $(3,0)(3,5)(2,10)$ & {$[867.37,13084.10,25300.80]$} \\
\hline 12 & $(3,0)(3,5)(1,10)$ & {$[1320.51,13723.90,26127.20]$} \\
\hline 13 & $(3,0)(2,5)(2,9)$ & {$[3339.13,14362.20,25385.40]$} \\
\hline 14 & $(3,0)(3,5)(2,10)$ & {$[1749.59,14958.10,28166.60]$} \\
\hline 15 & $(3,0)(3,5)(3,10)$ & {$[829.63,15480.30,30131.10]$} \\
\hline
\end{tabular}

TABLE IV

Dynamic Programming Results for Basic Example Problem

\section{The Optimal Replacement Policy}

As earlier we show the determination of the optimal replacement problem for the model example under fuzziness. Solving the functional equation given in Equation 7 for $N=15$ results in the optimal replacement policy $\Pi$ :

$$
(3,0)(3,5)(3,10)
$$

with

$$
F P V_{0.1}^{\star}(15)=[829.63,15480.30,30131.10] .
$$

This translates into buying asset 3 at time 0 , and again at time 5 and again at time 10 . In this solution, the modal values of each $F P V_{i}^{\star}(k)$ represent the $N P V_{i}^{\star}(k)$ of the deterministic model, while the lower and upper values indicate 
the overall uncertainty in the decision. The uncertainty signified by the width of the base of each $F P V_{i}^{\star}(k)$ also is equivalent to the range of possible values determined via multivariable sensitivity analysis. Thus a fuzzy model immediately provides both the traditional deterministic NPV for this policy as well as the range of values the NPV may take due to uncertainty in the parameters.

Other uncertain factors may be introduced via the relation function $f(a, n, C)$. If, for example, a moderate inflation increase of $[1 \%, 2 \%, 3 \%]$ per year were expected while the other parameters remained the same, the optimal replacement policy $\Pi$ becomes:

$$
(3,0)(2,5)(2,10)
$$

with

$$
F P V_{0.1}^{\star}(15)=[-969.74,16558.10,34313.60] .
$$

Contrast this with the traditional stochastic model SREDM where the solution is derived using Monte-Carlo simulation, a technique that generates a large number of realizations of the uncertain (and assumed random) variables and solves each set of them deterministically [31]. From this large sample, the probability that each alternative current asset is the optimal first choice can be estimated (see Figure 4) as well as the corresponding cumulative probability distributions of (1) the economic life of each current asset, (2) the NPV of the optimal sequence of challengers for a finite horizon (see Figure 5, and (3) the equivalent finite horizon time for infinite horizon problems.

We note that the probability distributions generated by

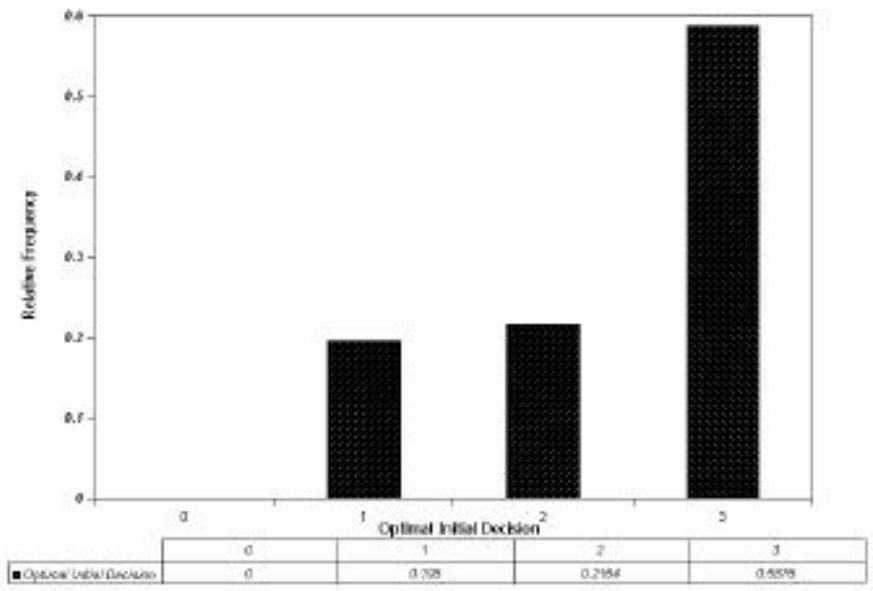

Fig. 4. Monte-Carlo simulation results for the example problem depicting the probability distribution for the optimal first choice.

SREDM are subjectively interpreted to determine the optimal current decision and no information regarding future decisions is available.

\section{Maintenance Decisions}

Not all replacement decisions are of the form where the only decisions are to "keep" or "replace" the existing asset. However, the DP formulation is sufficiently general to allow

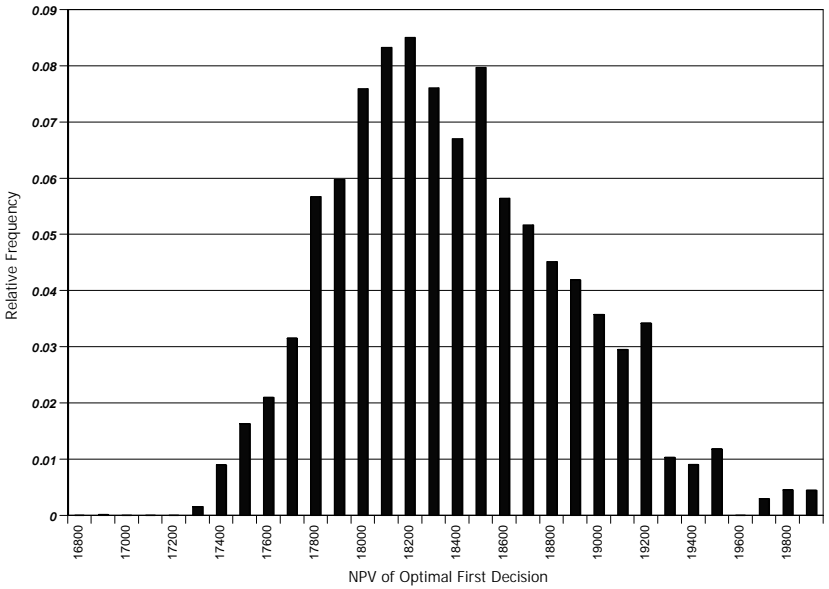

Fig. 5. Monte-Carlo simulation results for the example problem depicting the probability distribution for the optimal NPV.

for other options. For example, a third option may be the "purchase of a used machine". Bellman describes the DP formulation of the replacement model with the option to purchase a used machine as well as that of a model with an "overhaul" option [5]. The overhaul option can be done either in a general manner where the cash flows and other variables are functions of both the installation time and the overhaul time or by allowing the overhauled asset ( $t$ years old) to maintain the characteristics of a younger asset $\left(t^{\prime}<t\right.$ years old $)$.

The effects of the maintenance may also be uncertain variables that can be appropriately modeled as fuzzy numbers. Modeling an overhaul or maintenance option in PMSAR can be done either by defining functions that relate an overhauled asset's aggregate cash flows and salvage value to its installation time and overhaul time or by defining challenging assets that represent the costs and characteristics of an overhauled machine.

We return to the example problem for the PMSAR above to illustrate a maintenance option with an imprecise or vague effect. Suppose that the maintenance option is modeled as a "ghost" asset which is defined as follows: The purchase cost $P^{\prime}$ of the "ghost" asset $a^{\prime}$ is a function of the cost of the maintenance option on the asset $a$ currently in place and the number of years $n^{\prime}$ since $a$ was installed.

$$
P^{\prime}=\left([0.15,0.20,0.25] \cdot P_{a}\right)(1.03)^{n^{\prime}}
$$

where $P_{a}$ is the purchase price of asset $a$. This particular function states that the base maintenance cost is a fuzzy number that is $[15 \%, 20 \%, 25 \%]$ of the original cost of the asset with an increase of $3 \%$ per year since installation. The result of the maintenance is also fuzzy, a $[6 \%, 10 \%, 12 \%]$ reduction in the original operating costs for the following year. The component cash flows corresponding to this maintenance challenger $a^{\prime}$ are a function of the time $n^{\prime}$ that the maintenance occurs as well as the original asset and its installation time. Let us denote performing maintenance on the existing asset at period $n$ as $(M, n)$. The resulting optimal maintenance and replacement policy $\Pi$ for $N=15$ 
then becomes:

$$
\begin{aligned}
& (M, 0)(M, 1) \\
& (1,2)(M, 3)(M, 4)(M, 5)(M, 6) \\
& (1,7)(M, 8)(M, 9)(M, 10)(M, 11) \\
& (2,12)(M, 13)(M, 14)
\end{aligned}
$$

with an optimal fuzzy present value $F P V_{0.1}^{\star}(15)=$ [25709.00, 40749.90, 54887.80]. This translates to performing maintenance on the current defender at periods 0 and 1 , purchasing asset 1 at period 2 and performing maintenance on this asset each period until asset 1 is purchased again in time 7 . The maintenance at each period continues on asset 1 until asset 2 is purchased at period 12. Maintenance on asset 2 then performed at each remaining period. The fuzzy present value with this maintenance option, in this example, has risen significantly.

\section{Summary}

Probability theory has been used as the traditional approach for modeling uncertainty in economic analysis. This is acceptable only to the extent that uncertainty is satisfactorily equated with randomness. However, there exist other types of uncertainty that are especially relevant to economic decision analysis. Thus, there is a role to be played by nonprobabilistic uncertainty as shown in this effort. Many approaches have been shown possible. A brief survey of replacement analysis, focusing on the use of nonprobabilistic uncertainty, is given. The use of triangular fuzzy numbers provides a compromise between computational efficiency and realistic modeling of the uncertainty. Thus, this discussion emphasizes fuzzy numbers. In the extension to the economic life of an asset model, the uncertainty in the parameters is explicitly modeled. By only a three-fold increase in the number of computations, the optimal choice based on the decision maker's best estimates of these parameters is easily obtained. The traditional deterministic models are a special case of this new possibilistic model. In effect, PELAM performs multivariable sensitivity analysis on all the uncertain parameters concurrently and incorporates this uncertainty into the determination of the optimal decision. The benefits for PMSAR are virtually the same, except there is a greater increase in the number of computations due to the vertex method. Contrast this with the large number of repetitions that Monte-Carlo simulation requires, as well as the subjective interpretation of the results, and this disadvantage is not severe.

When probability distributions are not known, or when a stochastic model is too difficult to solve, fuzzy sets and possibility theory offer an efficient alternative in replacement analysis. There are a number of benefits for modeling the uncertainty in the replacement problem via fuzzy numbers. We outline a few of them:

1. The use of fuzzy uncertainty may be more appropriate when modeling systems with human subjectivity. The only existing technique in replacement analysis that modeled general uncertainty in the replacement decision was a Monte-Carlo simulation method [29].
2. Creating a triangular distribution from the best, worst, and most likely estimates of an expert is more appropriate for possibility theory than probability theory due to the lack of probabilistic information.

3. The results of each model can be easily plotted to provide a graphical representation of the effects of the uncertain parameters and can provide this information without a significant increase in the computational complexity. The algorithms are easily implemented on a personal computer.

4. The use of TFNs retains the triangular distribution throughout the solution algorithm and conveniently shows the best, worst, and most likely NPVs of each possible decision.

5. The use of fuzzy numbers corresponds to performing sensitivity analysis on all uncertain parameters simultaneously, as well as identifying the answer to the deterministic version of the problem which corresponds to the modal value of each fuzzy number.

We have illustrated the benefits of explicitly modeling uncertainty in engineering economy decisions using a fuzzy set-theoretic framework. As in traditional replacement analysis, the age of the asset served as the surrogate variable from which all cash flows could be determined. With all factors influencing the decision in the models being measured in terms of profit, the dynamic programming approach takes advantage of recurring subproblems to efficiently determine the optimal policy. Other factors such as operating efficiency, machine safety, and machine reliability may affect the replacement and maintenance decisions. Yet, these attributes are not easily quantified, especially in terms of profit. One approach in practice is to convert maintenance down-time into lost sales, examine safety issues in terms of insurance costs and worker's compensation, and use other obtainable measures as surrogates for these attributes. However, it is arguable that the decision making problem may not be adequately modeled in that framework. A fuzzy set-theoretic approach that identifies different measures for levels of repair, safety, maintenance up-time and down-time, and other imprecisely defined attributes may provide a more informative replacement policy. Modeling in this framework would require a multiple attribute decision making approach that incorporates the unquantifiable, incomplete, or nonobtainable information. The reader is referred to [26] for fuzzy approaches to multiple attribute and multiple objective decision making problems.

\section{REFERENCES}

[1] A.A. Alchian. Economic replacement policy. Technical Report Publication R-224, The RAND Corporation, Santa Monica, CA, 1952.

[2] J.C. Bean, J.R. Lohmann, and R.L Smith. A dynamic infinite horizon replacement economy decision model. The Engineering Economist, 30(2):99-120, Winter 1985.

[3] A.M. Behrens and F. Choobineh. Can economic uncertainty always be described by randomness? In Proceedings of the 1989 International Industrial Engineering Conference, pages 116-120, 1989.

[4] R.E. Bellman. Equipment replacement policy. SIAM Journal Applied Mathematics, 3:133-136, 1955. 
[5] R.E. Bellman and S.E. Dreyfus. Applied Dynamic Programming. Princeton University Press, Princeton, NJ, 1962.

[6] J. Bezdek. Fuzziness vs. probability - again (!?). IEEE Transactions on Fuzzy Systems, 2(1):1-3, 1994.

[7] G. Bortolan and R. Degani. A review of some methods for ranking fuzzy subsets. Fuzzy Sets and Systems, 15:1-19, 1985.

[8] J.J. Buckley. The fuzzy mathematics of finance. Fuzzy Sets and Systems, 21:257-273, 1987.

[9] J.J. Buckley. Solving fuzzy equations in economics and finance. Fuzzy Sets and Systems, 48:289-296, 1992.

[10] C.Y. Chiu and C.S. Park. Fuzzy cash flow analysis using present worth criterion. The Engineering Economist, 39(2):113-138, 1994.

[11] F. Choobineh and A. Behrens. Use of intervals and possibility distributions in economic analysis. Journal of the Operational Research Society, 43(9):907-918, 1992.

[12] W. Dong and H.C. Shah. Vertex method for computing functions of fuzzy variables. Fuzzy Sets and Systems, 14:65-78, 1987.

[13] S.E. Dreyfus and A.M. Law. The Art and Theory of Dynamic Programming. Academic Press, New York, 1977.

[14] D. Dubois and H. Prade. Operations on fuzzy numbers. International Journal of Systems Science, 9:612-626, 1978.

[15] D. Dubois and H. Prade. Fuzzy numbers: An overview. In J.C. Bezdek, editor, Analysis of Fuzzy Information, volume 1, pages 3-39. CRC Press, Boca Raton, FL, 1987.

[16] D. Dubois and H. Prade. Fuzzy sets-a convenient fiction for modeling vagueness and possibility. IEEE Transactions on Fuzzy Systems, 2(1):16-21, 1994.

[17] A.O. Esogbue and J. Kacprzyk. Fuzzy dynamic programming: A survey of main developments and applications. Archives of Control Sciences, 5(1-2):39-59, 1996.

[18] B. Gluss. An Elementary Introduction to Dynamic Programming: A State Equation Approach. Allyn and Bacon, Inc., 1972.

[19] C.P. Gupta. A note on the transformation of possibilistic information into probabilistic information for investment decisions. Fuzzy Sets and Systems, 56:175-182, 1993.

[20] W.E. Hearnes. Modeling with possibilistic uncertainty in the single asset replacement problem. In Proceedings of the International Joint Conference on Information Science, Fourth Annual Conference on Fuzzy Theory \& Technology, pages 552-555, Wrightsville Beach, NC, September 28 - October 11995.

[21] J. Kacprzyk. Decision-making in a fuzzy environment with fuzzy termination time. Fuzzy Sets and Systems, 1:169-179, 1978

[22] A. Kaufmann and M.M. Gupta. Fuzzy Mathematical Models in Engineering and Management Science. Elsevier Science Publishers, B.V., 1988.

[23] G.J. Klir. On the alleged superiority of probabilistic representation of uncertainty. IEEE Transactions on Fuzzy Systems, 2(1):27-31, 1994.

[24] G.J. Klir and B. Yuan. Fuzzy Sets and Fuzzy Logic: Theory and Applications. Prentice Hall PTR, Upper River Saddle, NJ, 1995.

[25] B. Kosko. The probability monopoly. IEEE Transactions on Fuzzy Systems, 2(1):32-33, 1994.

[26] Y.-J. Lai and C.-L. Hwang. Fuzzy Multiple Objective Decision Making. Springer-Verlag, Berlin, 1994.

[27] M. Laviolette and J.W. Jr. Seaman. The efficacy of fuzzy representations of uncertainty. IEEE Transactions on Fuzzy Systems, 2(1):4-15, 1994.

[28] M. Li Calzi. Towards a general setting for the fuzzy mathematics of finance. Fuzzy Sets and Systems, 35:265-280, 1990.

[29] J.R. Lohmann. A stochastic replacement economic decision model. IIE Transactions, 18:182-194, 1986.

[30] R.V. Oakford. Capital Budgeting: A Quantitative Evanluation of Investment Alternatives. The Ronald Press Co., New York, 1970.

[31] R.V. Oakford, J.R. Lohmann, and A. Salazar. A dynamic replacement economy decision model. IIE Transactions, 16:65-72, 1984.

[32] R.V. Oakford, A. Salazaar, and H.A. DiGiulio. The long term effectiveness of expected net present value maximization in an environment of incomplete and uncertain information. AIIE Transactions, 13:265-276, 1981.

[33] R.J. Samuelson. Imperfect vision: A flawed cpi makes it harder for us to read the past and plan for the future. Newsweek, 55, 1996.

[34] L. Savage. The Foundations of Statistics. Wiley, New York, 1954.
[35] G.P. Sharp-Bette and C.S. Park. Advanced Engineering Economics. John Wiley and Sons, Inc., 1990.

[36] G. Terborgh. Dynamic Equipment Policy. Machinery and Applied Products Institute, Washington, DC, 1949.

[37] A. Tversky and D. Kahneman. Belief in the law of small numbers. Psychological Bulletin, 2:105-110, 1971.

[38] A. Tversky and D. Kahneman. Judgement under uncertainty: Heuristics and biases. Science, 185:1124-1131, 1974.

[39] A. Tversky and D. Kahneman. The framing of decisions and the psychology of choice. Science, 211:3-8, 1981.

[40] J. Von Neumann and O. Morgenstern. Theory of Games and Economic Behavior. Princeton University Press, Princeton, NJ, 1944.

[41] H.M. Wagner. Principles of Operations Research. Prentice-Hall, 1975.

[42] T.L. Ward. Discounted fuzzy cash flow analysis. In Proceedings of the 1985 Fall Industrial Engineering Conference, pages 476$481,1985$.

[43] L.A. Zadeh. Fuzzy sets. Information and Control, 8:338-353, 1965.

[44] L.A. Zadeh. The concept of a linguistic variable and its application to approximate reasoning i, ii, iii. Information Sciences, 8-9:8:199-251; 8:301:357; 9:43-80, 1975.

[45] L.A. Zadeh. Fuzzy sets as a basis for a theory of possibility. IEEE Transactions on Systems, Man, and Cybernetics, 1:3-28, 1978 .

[46] H.J. Zimmerman. Fuzzy Set Theory and Its Applications. Kluwer Academic Press, Boston, MA, second edition, 1991.

\section{APPENDIX}

Fuzzy set theory, a generalization of classical set theory, was developed in 1965 [43]. Fuzziness describes sets that have no sharp transition from membership to nonmembership. Traditional modeling methods assume certain and unambiguous structures and parameters, but uncertainty is inherent in most real-world systems. Fuzzy set theory provides a strict mathematical theory to describe this inherent characteristic.

Let $\mathbf{X}$ be a collection of objects denoted generically by $\mathrm{x}$.

Definition 1 ([43]) A fuzzy set (or fuzzy subset) $\tilde{A}$ in $\mathbf{X}$ is a set of ordered pairs

$$
\tilde{A}=\left\{\mathbf{x}, \mu_{\tilde{A}}(\mathbf{x}) \mid \mathbf{x} \in \mathbf{X}\right\}
$$

where $\mu_{\tilde{A}}(\mathbf{x})$ is the membership function of $\mathbf{x}$ in $\tilde{A}$ which maps $\mathbf{X}$ to the membership space $\mathbf{M}$. If $\mathbf{M}$ is the closed interval $[0,1]$, then $\tilde{A}$ is called a normal fuzzy set.

Definition 2 ([43]) The $\alpha$-level set of a fuzzy set $\tilde{A}$ is the crisp set of elements that belong to $\tilde{A}$ at least to the degree $\alpha \in[0,1]$.

Definition 3 ([43]) A fuzzy set $\tilde{A}$ is convex if

$$
\mu_{\tilde{A}}\left(\lambda \mathbf{x}_{1}+(1-\lambda) \mathbf{x}_{2}\right) \geq \min \left(\mu_{\tilde{A}}\left(\mathbf{x}_{1}\right), \mu_{\tilde{A}}\left(\mathbf{x}_{2}\right)\right)
$$

for $\mathbf{x}_{1}, \mathbf{x}_{2} \in \mathbf{X}$ and $\lambda \in[0,1]$. An alternative definition is a fuzzy set is convex if all $\alpha$-level sets are convex.

The basis for generalizing crisp (non-fuzzy) mathematical concepts to fuzzy sets is the extension principle.

Definition 4 ([44]) Let $\mathbf{X}$ be a Cartesian product of universes $\mathbf{X}=\mathbf{X}_{1}, \ldots, \mathbf{X}_{r}$, and $\tilde{A}_{1}, \ldots, \tilde{A}_{r}$ be $r$ fuzzy sets in $\mathbf{X}_{1}, \ldots, \mathbf{X}_{r}$, respectively. Let $f$ be a mapping from $\mathbf{X}$ to a universe $\mathbf{Y}, y=f\left(\mathbf{x}_{1}, \ldots, \mathbf{x}_{r}\right)$. The extension principle allows the definition of a fuzzy set $\tilde{B}$ in $\mathbf{Y}$ by

$$
\tilde{B}=\left\{\left(y, \mu_{\tilde{B}}(y)\right) \mid y=f\left(\mathbf{x}_{1}, \ldots, \mathbf{x}_{r}\right),\left(\mathbf{x}_{1}, \ldots, \mathbf{x}_{r}\right) \in \mathbf{X}\right\}
$$


where $\mu_{\tilde{B}}(y)$ is

$$
\sup _{\left(\mathbf{x}_{1}, \ldots, \mathbf{x}_{r}\right) \in f^{-1}(y)} \min \left\{\mu_{\tilde{A}_{1}}\left(x_{1}\right), \ldots, \mu_{\tilde{A}_{r}}\left(x_{r}\right)\right\}
$$

if $f^{-1}(y) \neq \emptyset$ and 0 otherwise.

Fuzzy numbers are a particular kind of fuzzy set.

Definition 5 ([46]) A fuzzy number $\tilde{M}$ is a convex normal fuzzy set on the real line $R$ such that (1) there exists exactly one $\mathbf{x}_{0} \in R$ with $\mu_{\tilde{M}}(\mathbf{x})=1$ and $(2) \mu_{\tilde{M}}(\mathbf{x})$ is piecewise continuous. Denote the $\mathbf{x}_{0} \in R$ that satisfies (1) as the modal value of the fuzzy number.

Fuzzy arithmetic is based on the extension principle. The arithmetic operations of addition, subtraction, multiplication, and division were developed by Dubois and Prade [14] and an excellent overview is given in [15].

Definition $6([14])$ A fuzzy number $\tilde{M}$ is positive (negative) if $\mu_{\tilde{M}}(\mathbf{x})=0$ for all $x<0(x>0)$.

Though the addition, subtraction, multiplication, and division operations are defined for general fuzzy numbers, we focus on the operations as they apply to triangular fuzzy numbers. Let $\tilde{M}_{1}=\left[l_{1}, m_{1}, u_{1}\right]$ and $\tilde{M}_{2}=\left[l_{2}, m_{2}, u_{2}\right]$ be two triangular fuzzy numbers with respective lower, most likely, and upper estimates. The sum is defined as

$$
\tilde{M}_{1}+\tilde{M}_{2}=\left[l_{1}+l_{2}, m_{1}+m_{2}, u_{1}+u_{2}\right]
$$

and is associative and commutative. The subtraction operation is simply the addition operation on two fuzzy numbers, one of which has been multiplied by the scalar -1 . Scalar multiplication for TFNs is

$$
a \tilde{M}_{1}= \begin{cases}{\left[a l_{1}, a m_{1}, a u_{1}\right]} & \text { for } a \geq 0 \\ {\left[a u_{1}, a m_{1}, a l_{1}\right]} & \text { for } a<0\end{cases}
$$

The difference of two TFNs is therefore

$$
\tilde{M}_{1}-\tilde{M}_{2}=\left[l_{1}-u_{2}, m_{1}-m_{2}, u_{1}-l_{2}\right]
$$

which is also associative and commutative.

The multiplication operation for TFNs is only weakly distributive over addition. Therefore the solution procedure must either use the vertex method [12] or it may give a different outcome. For example, the following property holds true in all cases

$$
\tilde{M}_{1}\left(\tilde{M}_{2}+\tilde{M}_{3}\right) \subseteq \tilde{M}_{1} \tilde{M}_{2}+\tilde{M}_{1} \tilde{M}_{3}
$$

and is only equality under special circumstances or through the use of the vertex method. The vertex method for TFNs is straightforward: Each of the three TFNs above has 2 extreme points, $l_{i}$ and $u_{i}$ for $i=1,2,3$. All $2^{3}$ combinations are calculated for the above expression and the minimum and maximum values are chosen. The drawback is that there is an exponential number of calculations under this algorithm.

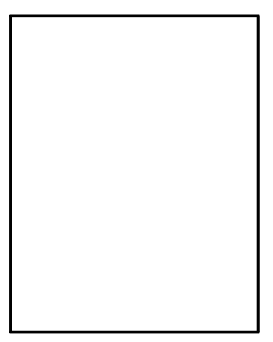

Augustine O. Esogbue received a B.S. degree in electrical engineering and mathematics from the University of California, Los Angeles, in 1964, a M.S. degree in industrial engineering and operations research from Columbia University, New York, in 1965, and a Ph.D. in industrial and systems engineering from the University of Southern California, Los Angeles, in 1968. He served as an Assistant Professor in the Department of Operations Research and Systems Research at Case Western Reserve University from 1968 to 1972 . He then joined the faculty of the Department of Industrial and Systems Engineering at the Georgia Institute of Technology as an Associate Professor in 1972 and was promoted to Professor in 1977. He is currently the Director of the Intelligent Systems and Controls Laboratory at Georgia Tech. His research interests include dynamic programming, fuzzy sets, decision making and control in a fuzzy environment, and operations research with application to socio-technical problems such as health care, water resource management, and disaster control planning.

Dr. Esogbue is active on the program committees for international conferences and serves on the editorial boards of several journals, including the International Journal of Computer Science and Its Applications, Journal of Mathematical Analysis and Applications, and Fuzzy Sets and Systems. He also actively promotes the National Society of Black Engineers and is a member of its National Advisory Board. in general engineering from the United States Military Academy at West Point, New York, in 1989 . He is currently a Ph.D. candidate in the Department of Industrial and Systems Engineering at the Georgia Institute of Technology in Atlanta, Georgia. His current research interests include engineering economy, decision analysis, dynamic programming, and intelligent control. 